\title{
Prospects of a search for a new massless neutral gauge boson at the ILC
}

\author{
E. Boos, ${ }^{1}$ V. Bunichev, ${ }^{1}$ and H. J. Schreiber ${ }^{2}$ \\ ${ }^{1}$ Skobeltsyn Institute of Nuclear Physics, MSU, 119992 Moscow, Russia \\ ${ }^{2}$ DESY, Deutsches Elektronen-Synchrotron, D-15738 Zeuthen, Germany
}

(Received 25 November 2007; published 11 July 2008)

\begin{abstract}
Prospects to search for a new massless neutral gauge boson, the paraphoton, in $e^{+} e^{-}$collisions at center-of-mass energies of 0.5 and $1 \mathrm{TeV}$ are studied. The paraphoton naturally appears in models with Abelian kinetic mixing. A possible realistic model-independent lowest order effective Lagrangian contains magnetic interactions of the paraphoton with the standard model fermion fields. These interactions are proportional to the fermion mass and grow with energy, with however very weak paraphoton couplings to ordinary matter. At the ILC, a potentially interesting process to search for the paraphoton is its radiation from top quarks, so that the event topology of interest is a pair of acoplanar top quarks decaying to jets and missing energy. By combining many discriminating features of signal and background events, efficient paraphoton event selection is achieved, allowing us to set limits for the top-paraphoton coupling. Arguments in favor of the missing energy as the paraphoton with spin 1 are discussed.
\end{abstract}

DOI: 10.1103/PhysRevD.78.015007

PACS numbers: $12.60 . \mathrm{Cn}$

\section{INTRODUCTION}

Modern elementary particle field theories are based on the principle of gauge invariance. This means that the Lagrangian of the theory should be invariant with respect to group transformation of the local symmetry, which leads to a corresponding number of massless vector gauge boson fields. In the standard model (SM), based on the $U_{Y}(1) \times$ $S U_{L}(2) \times S U_{C}(3)$ gauge symmetry group, 12 gauge vector bosons exist. Three of them, the electroweak bosons $W^{ \pm}$ and $Z^{0}$, get masses due to the Higgs mechanism of spontaneous symmetry breaking. The eight massless strongly interacting gauge bosons, the gluons, are confined in hadrons and only one directly observed massless neutral vector boson, the well-known photon, exists within the SM.

Although the standard model does not require any additional gauge fields, it is possible to introduce gauge invariant operators in the Lagrangian that involve new gauge fields not forbidden by the basic principle of gauge invariance. An example is given in Ref. [1] by the Abelian kinetic mixing of the SM $U_{Y}(1)$ field with a new $U_{P}(1)$ field in a gauge invariant manner. The mixing term of the two $U(1)$ fields can be diagonalized and canonically normalized by an $S L(2, R)$ transformation in a way that one linear combination of the fields corresponds to the ordinary photon that couples in the usual manner to all electrically charged particles within the SM. The other linear combination appears as a massless spin-1 neutral particle, referred to as the "paraphoton" in Ref. [2] and denoted by $\gamma^{\prime}$ in this paper. This mechanism also provides an elegant way of introducing millicharged particles ${ }^{1}$ into the theory [3].

\footnotetext{
${ }^{1}$ Millicharged particles are related to fields charged under the $U_{P}(1)$ group. Interaction of these particles with SM fields should be very small and proportional to the kinetic mixing parameter.
}

The paraphoton couples directly to millicharged fermions and only indirectly to the SM fields via higher massdimension operators.

In this study, we follow an approach proposed in Ref. [4] where the effective Lagrangian of the interaction of the paraphoton with the SM fermion fields was proposed, by considering higher dimensional operators. A possible lowest order Lagrangian that preserves both the new $U_{P}(1)$ and the SM gauge symmetries with the SM fermion chirality structure has the following form:

$$
\begin{aligned}
& \frac{1}{M^{2}} P_{\mu \nu}\left(\bar{q}_{L} \sigma^{\mu \nu} C_{u} \tilde{H} u_{R}+\bar{q}_{L} \sigma^{\mu \nu} C_{d} H d_{R}+\bar{l}_{L} \sigma^{\mu \nu} C_{e} H e_{R}\right. \\
& \quad+\text { H.c. }),
\end{aligned}
$$

where $q_{L}, l_{L}$ are the quark and lepton doublets, $u_{R}, d_{R}$ the up- and down-type $S U(2)$ singlet quarks, $e_{R}$ the electrically-charged $S U(2)$-singlet leptons, and $H$ is the Higgs doublet. An index labeling the three fermion generations is implicit here. The $3 \times 3$ matrices in flavor space, $C_{u}, C_{d}, C_{e}$, have dimensionless complex elements, and $M$ is the mass scale where the operators are generated.

One can see that the interactions of the paraphoton with standard model fermions are suppressed by two powers of the mass scale $M$, but are directly proportional to the fermion mass $m_{f}$ and the dimensionless coupling strength parameter $C_{f}$, with $f=u, d, e$. The coefficients $C_{f}$ are unknown, but various phenomenological constraints exist. In particular, limits on these parameters for light fermions were, for example, deduced from paraphoton annihilation to muon pairs, $\gamma^{\prime} \gamma \rightarrow \mu^{+} \mu^{-}$, or the Comptonlike process, $\gamma^{\prime} \mu^{ \pm} \rightarrow \gamma \mu^{ \pm}$, assuming the $\gamma^{\prime}$ interaction rate equals the expansion rate of the universe at freeze out. Together with successful predictions of primordial nucleon-synthesis the $\mu^{-} \mu^{+} \gamma^{\prime}$ coupling parameter is 
bounded to $M / \sqrt{c_{\mu}} \geqslant 1.5 \mathrm{TeV}$, where $c_{\mu}$ is related to $C_{\mu}$ via $c_{f}=C_{f} v_{h} /\left(\sqrt{2} m_{f}\right)$, and $v_{h}$ is the vacuum expectation value of the Higgs field. In other words, star cooling by $\gamma^{\prime}$ emission constraints the electron-paraphoton interaction since the associated energy loss is proportional to the square of $4 c_{e} m_{e}^{2} / M^{2}$. The limit on $\gamma^{\prime}$ emission through Bremsstrahlung, such as $e^{-}+{ }^{4} \mathrm{He} \rightarrow e^{-}+{ }^{4} \mathrm{He}+\gamma^{\prime}$, from the core of red giant stars [5] requires $M / \sqrt{c_{e}} \gtrsim$ 3.2 TeV, while Compton-like scattering, $\gamma e^{-} \rightarrow \gamma^{\prime} e^{-}$, in horizontal-branch stars sets a somewhat weaker limit of $M / \sqrt{c_{e}} \geq 1.8 \mathrm{TeV}$. A constraint on the $\gamma^{\prime}$-coupling to nucleons of $M / \sqrt{c_{N}} \geqslant 7 \mathrm{TeV}$ has been estimated from the neutrino signal of the supernova 1987A assuming the supernova was cooled predominantly by neutrinos. More details of possible lower limits on $\gamma^{\prime}$ interactions with fermions are discussed in Ref. [4].

An intriguing aspect of the presence of an additional gauge field like the paraphoton is the possible existence of fields charged under the $U_{P}(1)$ group. Simple renormalizable models generate operators, see Eq. (1), which are associated with new heavy states. The lightest particle of this type with negligible electrical charge is stable and could be a viable dark matter candidate.

As follows from the Lagrangian in Eq. (1) and existing bounds on the couplings $c_{f}$ for light fermions, the paraphoton couples most strongly to the top quark and very weakly to light fermions. Therefore, one expects the most interesting process to search for the paraphoton to be $\gamma^{\prime}$ radiation from the top. Since so far no constraint on $c_{t}$ exists, access to $M / \sqrt{c_{t}}$ seems possible and corresponding limits might be set for the first time. It seems a priori difficult to perform $\gamma^{\prime}$ searches at hadron colliders because of very large $t \bar{t}+$ multijet background. The next generation $e^{+} e^{-}$linear collider (ILC) provides potentially a possibility to search for the paraphoton via the channel

$$
e^{+} e^{-} \rightarrow t \bar{t} \gamma^{\prime}
$$

The paper is organized as follows. In Sec. II, basic properties of signal events are studied at the parton level in order to extract information that might be helpful to discriminate signal from background events. Section III describes the search strategy for the $\gamma^{\prime}$ based on hadronic $W$ boson decays, $W \rightarrow q \bar{q}$, to avoid complications from leptonic $W$ decays with neutrinos in the final state, also carrying energy away. The analysis is performed based on full simulation including ILC detector response. Section IV discusses accessible limits on $M / \sqrt{c_{t}}$ from an excess of signal events over the SM background expectations and gives arguments in favor of the nature of the paraphoton. Conclusions are summarized in Sec. V.

\section{THE SIGNAL REACTION $e^{+} e^{-} \rightarrow \boldsymbol{t} \bar{t} \gamma^{\prime}$}

A parameter scan was done for various couplings and detailed simulations of $t \bar{t} \gamma^{\prime}$ signal events were performed for a "reasonable" value of the effective coupling parameter $M / \sqrt{c_{t}}\left(M / \sqrt{c_{t}}=0.2 \mathrm{TeV}\right)$ when the signal is large enough to be clearly distinguishable from the SM background. All the computations and simulations were performed with the present design for the ILC [6], collision energies $\sqrt{s}=0.5$ and $1.0 \mathrm{TeV}$ and integrated luminosity of 0.5 and $1 \mathrm{ab}^{-1}$, respectively. The characteristics of the signal reaction were computed and partonic events were generated using the program package COMPHEP [7]. The Feynman rules for the fermion-fermion- $\gamma^{\prime}$ vertices following from the effective Lagrangian (1)

$$
\frac{c_{f}}{M^{2}} \cdot m_{f} \cdot p_{\nu}^{\gamma^{\prime}}\left(\gamma^{\nu} \gamma^{\mu}-\gamma^{\mu} \gamma^{\nu}\right)
$$

have been implemented into COMPHEP. An interface with PYTHIA 6.202 [8] allows one to simulate initial- and finalstate radiation and jet hadronization. Also, beamstrahlung effects [9] are taken into account. The signal event rates for both energies are given in Table I. Simulations were performed for $M / \sqrt{c_{t}}=0.2 \mathrm{TeV}$ enabling sufficient $\gamma^{\prime}$ events at both energies. It is worthwhile to mention that the effective interaction Lagrangian approach cannot be applied for $M / \sqrt{c_{t}} \leqq 0.1 \mathrm{TeV}$ since the effective coupling parameter $\frac{c_{t} m_{\mathrm{top}} E}{M^{2}}$ gets too large for typical collision energies $E$.

In order to establish a search strategy for the paraphoton in $t \bar{t}$ events, it is advantageous to know whether an off-shell or on-shell top quark radiates the $\gamma^{\prime}$. Figure 1 shows the

TABLE I. $t \bar{t} \gamma^{\prime}$ event rates at $\sqrt{s}=0.5$ and $1 \mathrm{TeV}$ and an integrated luminosity of 0.5 and $1 \mathrm{ab}^{-1}$, respectively.

\begin{tabular}{lcc}
\hline \hline$M / \sqrt{c_{t}}[\mathrm{TeV}]$ & $\sqrt{s}=0.5 \mathrm{TeV}$ & $\sqrt{s}=1 \mathrm{TeV}$ \\
\hline 0.2 & 5700 & 42500 \\
\hline \hline
\end{tabular}

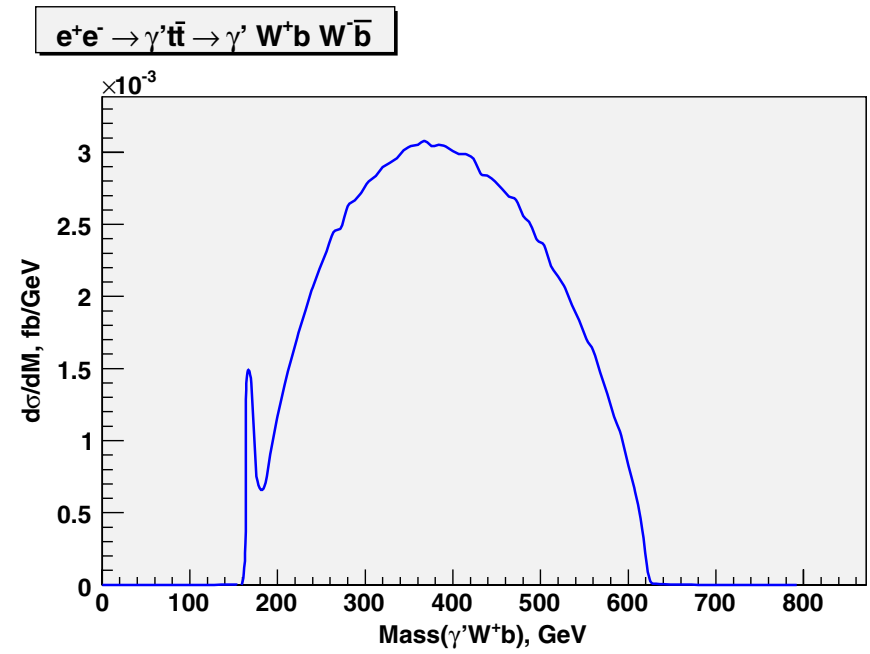

FIG. 1 (color online). Invariant mass of the $\gamma^{\prime} W b$ system. 

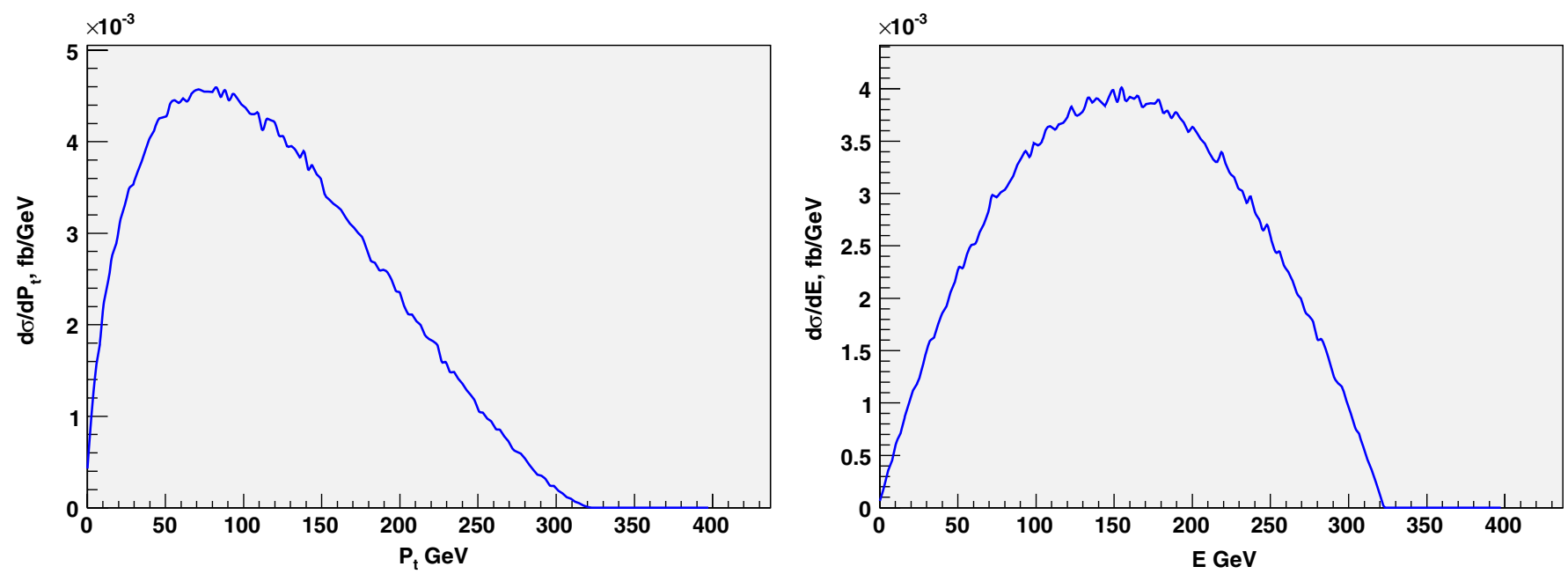

FIG. 2 (color online). $\quad \gamma^{\prime}$ transverse momentum (left) and energy (right) distributions at $\sqrt{s}=1 \mathrm{TeV}$.

invariant mass of the $\gamma^{\prime} W b$ system for the top quark that radiates the paraphoton. Clearly, in most cases the paraphoton is radiated from a top quark being off-shell, and $\gamma^{\prime}$ search strategies should be based on on-shell top with $t \rightarrow$ $W b$ decays in association with the $\gamma^{\prime}$. The energy and transverse momentum distributions of the $\gamma^{\prime}$ shown in Fig. 2 reflect that the paraphoton-top coupling is proportional to the paraphoton momentum, so that large missing energy, $\mathbb{E}$, can be used to identify signal events.

\section{SIGNAL EVENT SELECTION}

After event generation using COMPHEP, PYTHIA, and the COMPHEP-PYTHIA interface packages with the Les Houches Accord implemented [10] an approximate response of an ILC detector was simulated by means of SIMDET_v4 [11].

There are a number of SM background sources that have similar or identical final-state signatures, i.e., a signature of $t \bar{t}+\mathbb{E}_{T}$ with acoplanar top quarks decaying to jets and missing energy. The most important background consists of $t \bar{t}+(\gamma)$ events, where photons from initial state radiation (ISR) are not detected. The event rates expected for both energies are given in Table II. They exceed substantially the number of signal events (see Table I) for the chosen value of the parameter $M / \sqrt{c_{t}}$. The next significant background to consider is $e^{+} e^{-} \rightarrow t \bar{t}+\nu \bar{\nu}$, with the same signature as for the signal. The corresponding event rates are also given in Table II. An invariant mass cut of e.g., $M_{\nu \bar{\nu}}<80 \mathrm{GeV}$, i.e. a cut on the event missing mass, removes most $t \bar{t} \nu \bar{\nu}$ events.

TABLE II. Background events at $\sqrt{s}=0.5$ and $1 \mathrm{TeV}$ for integrated luminosity of 0.5 and $1 \mathrm{ab}^{-1}$, respectively.

\begin{tabular}{lcc}
\hline \hline Background & $\sqrt{s}=0.5 \mathrm{TeV}$ & $\sqrt{s}=1 \mathrm{TeV}$ \\
\hline$t \bar{t}(\gamma)$ & 276675 & 200310 \\
$t \bar{t} \nu \bar{\nu}$ & 75 & 930 \\
\hline \hline
\end{tabular}

In a first attempt to establish an excess of paraphoton events over the background, a conventional method was applied by using consecutive cuts on kinematic variables based on either the energy flow objects or, utilizing a jet finder, the 4-momenta of jets consistent with the $t \bar{t} \rightarrow$ $(W b)(W b) \rightarrow(q \bar{q}) b(q \bar{q}) b$ decay chain. The variables used may be classified into three categories: global event kinematics, variables based on jet properties and variables based on jet correlations. We considered the missing event energy, $\not{E}$, missing transverse energy, $\not{E}_{T}$, missing momentum, $\not p$, missing transverse momentum, $\not \not_{T}$, the event aplanarity, thrust, missing mass squared, the angle between the top momenta, and the coplanarity angle (the angle between the beam, the $t$ and $\bar{t}$ ) as well as, for a given hemisphere, the largest energy of jets and the largest angle between two jets. Jets were reconstructed by means of the routine PUCLUS from PYTHIA which relies on a cluster analysis method using particle momenta. The "jet-resolutionpower" was adjusted to provide 7- and 8-jet event rates in accord with expectations from gluon radiation. The method of consecutive cuts, however, was found to be
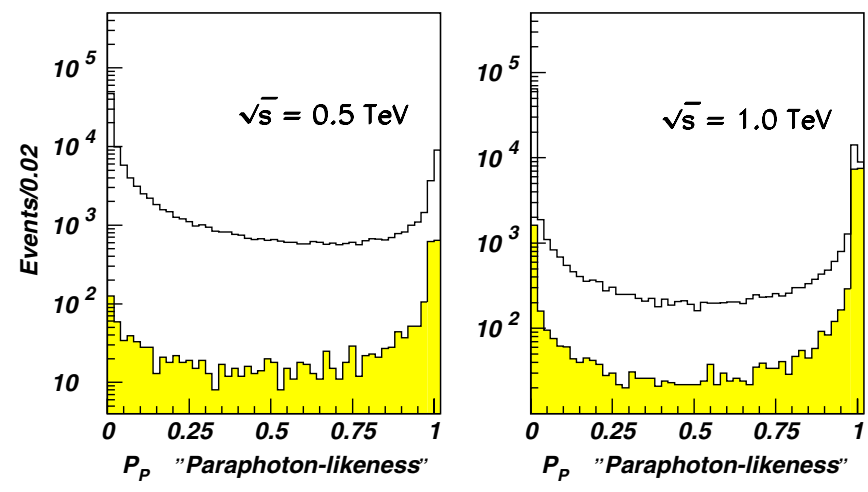

FIG. 3 (color online). Distributions of the discriminant variable $P_{P}$ for $t \bar{t} \gamma^{\prime}$ signal events (shaded) and the sum of signal and background events at $\sqrt{s}=0.5$ (left) and $1 \mathrm{TeV}$ (right). 

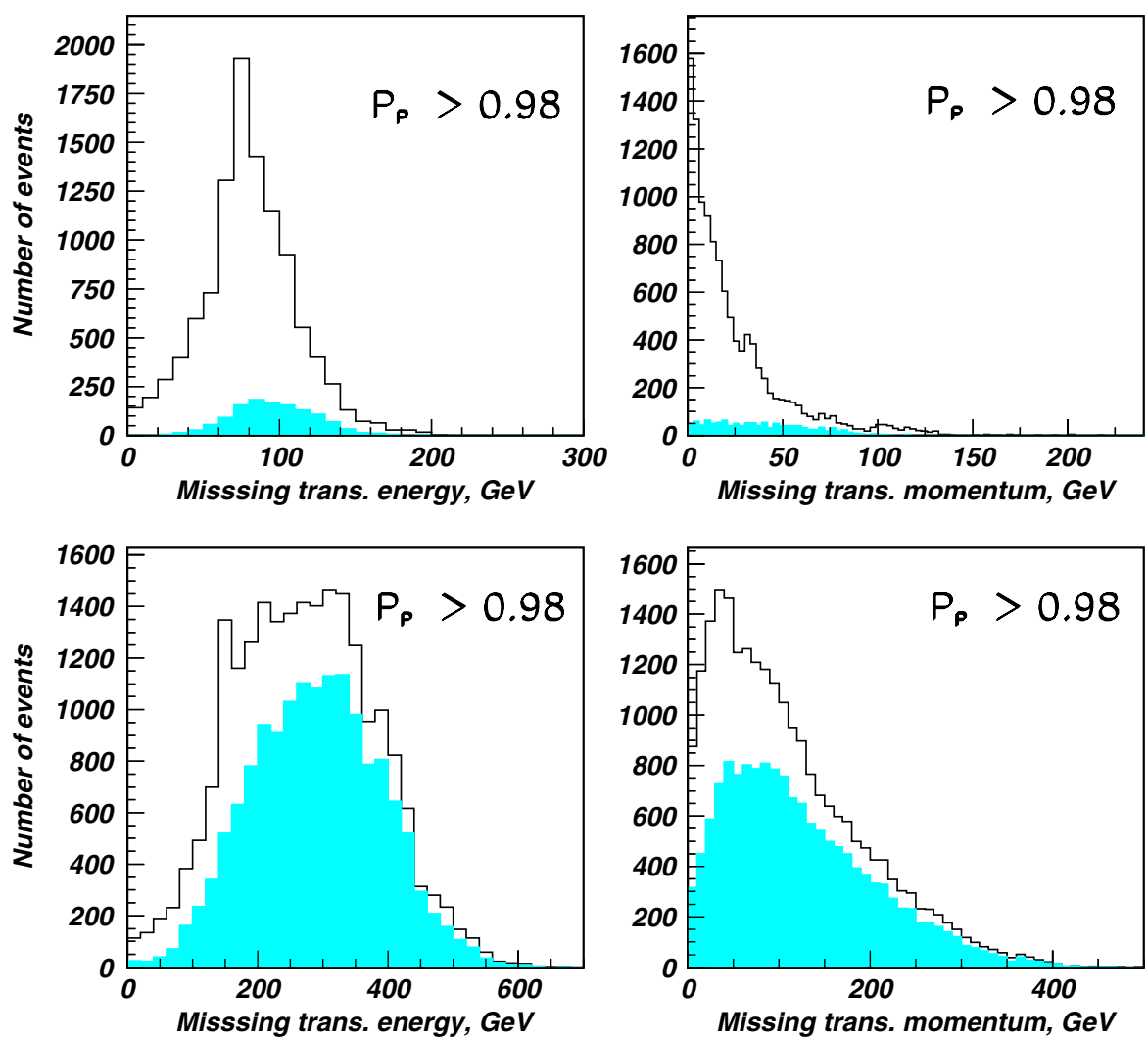

FIG. 4 (color online). $\quad \mathscr{E}_{T}$ and $\not \not_{T}$ distributions of $t \bar{t} \gamma^{\prime}$ signal events (shaded) and the sum of signal and background events at $\sqrt{s}=0.5$ (top) and $1 \mathrm{TeV}$ (bottom).

inefficient to select signal from background because of a lack of distinct properties between signal and background events.

In cases of large background, small signal event rates, and of variables with only small discrimination power, one needs to pursue more sophisticated strategies to extract the signal. We used a multivariate technique based on the likelihood method to select signal events. Kinematic variables as discussed above were combined into a global discriminant variable $P_{P}$, designed to give a measure of the "Paraphoton-likeness" of any particular event. This quantity was constructed from the variables after normalization based on large statistics samples of simulated signal and background events. For each event and variable $i$, signal and background probabilities $\left(P_{S}^{i}\right.$ and $\left.P_{B}^{i}\right)$ were then calculated, and by multiplication of all signal probabilities ( $\prod_{i=1}^{n} \frac{P_{S}^{i}}{P_{S}^{i}+P_{B}^{i}}, i=1 \ldots n$, with $n=18$, the number of variables taken into account) the sensitivity for an event to be a paraphoton candidate was maximized. The quantity so obtained was constrained to lie in the region $[0 ; 1]$. The background events are preferentially distributed at low $P_{P}$ values while for signal events $P_{P}$ is expected to be concentrated close to 1 . The distributions of $P_{P}$ for both center-of-mass energies are shown in Fig. 3. Clear accumulations of $\gamma^{\prime}$ candidate events can be recognized near $P_{P}=1$, with some non-negligible background, in particu- lar, at $0.5 \mathrm{TeV}^{2}$ By choosing an optimal cut value for the discriminant $P_{P}$ one gets a signal selection efficiency of $49 \%(76 \%)$ at $\sqrt{s}=0.5(1) \mathrm{TeV}$, while only $9 \%$ of background events survive. Figure 4 shows the $\not_{T}$ and $\not \not_{T}$ distributions at 0.5 and $1 \mathrm{TeV}$ for the signal (shaded) and the sum of signal and background events, surviving the $P_{P}$ cut. At $\sqrt{s}=0.5 \mathrm{TeV}, S / \sqrt{B}=12$ for $M / \sqrt{c_{t}}=0.2 \mathrm{TeV}$, while $S / \sqrt{B}=163$ at $1 \mathrm{TeV}$, i.e., the probability of measuring the signal event rates as a result of a background fluctuation is $0.5 \times 10^{-12}$ and $<10^{-15}$ at 0.5 and $1 \mathrm{TeV}$, respectively, using Gaussian sampling of uncertainties. In this way, a fairly background-free signal event sample can be extracted for further measurements at $1 \mathrm{TeV}$. The situation is much less convenient at $0.5 \mathrm{TeV}$.

\section{DISCUSSION OF THE RESULTS}

If an excess of signal events over the SM background is established, limits on the inverse coupling parameter $M / \sqrt{c_{t}}$ accessible for a significance of $S / \sqrt{B}=5$ can be derived. We consider this figure as sufficient for the para-

\footnotetext{
${ }^{2}$ The peak at $P_{P} \simeq 0$ for signal events is due to $\gamma^{\prime}$ events where for at least one variable $i$ the signal probability, $P_{S}^{i}$, is very close to zero, so that an overall paraphoton probability close to zero is obtained. Such imperfect selection resembles the occasionally small discriminating power of the variables used.
} 
photon discovery. The numbers of surviving $\gamma^{\prime}$ events for $5 \sigma$ discovery at $0.5(1.0) \mathrm{TeV}$ energies and an integrated luminosity of $0.5(1.0) \mathrm{ab}^{-1}$ can be converted into limits for $M / \sqrt{c_{t}}$. These limits are found to be 0.33 and $0.61 \mathrm{TeV}$ for 0.5 and $1 \mathrm{TeV}$, respectively. The $1 \mathrm{TeV}$ value of $M / \sqrt{c_{t}}=$ $0.61 \mathrm{TeV}$ is expected to be the most stringent limit accessible at the ILC.

The signal-to-background ratio, $S / B$, of about 1.8 at $1 \mathrm{TeV}$ is sufficiently large to probe the spin assignment of the $\gamma^{\prime}$. In order to demonstrate its spin-1 nature, we follow studies performed to establish the vector nature of the gluon in 3-jet $e^{+} e^{-}$annihilation events at PETRA [12-15] and LEP [16-18] energies, based on predictions that a spin $-\frac{1}{2}$ quark radiates the spin- 1 gluon. Many observables have been measured, including the Ellis-Karlinger angular distribution [19], energy-energy correlations [20], and jet masses [21], as well as the three-jet [22] and multijet production cross sections [23]; all of which were important in establishing the properties of the gluon, in particular, its spin.

For the sake of demonstration, we assign for each $1 \mathrm{TeV}$ signal event candidate with $\mathbb{E}_{T}>330 \mathrm{GeV}$ the fractional energy variables $x_{i}=E_{i} / E_{b}(i=1,2,3)$ to the $t, \bar{t}$, and $\gamma^{\prime}$, with $E_{b}$ the nominal incident beam energy. After ordering the $x_{i}$ such that $x_{1} \geq x_{2} \geq x_{3}$ and assuming the top quark mass is small with respect to $E_{b}$, a Lorentz boost is performed that brings the two less energetic jets to their c.m. frame where they should emerge back-to-back. The angle which these jets make with the thrust axis is defined as the Ellis-Karliner angle $\theta_{\mathrm{EK}}[19]$

$$
\cos \theta_{\mathrm{EK}}=\frac{x_{2}-x_{3}}{x_{1}}=\frac{\sin \theta_{2}-\sin \theta_{3}}{\sin \theta_{1}},
$$

where $\theta_{i}$ is the angle between the two jets opposite to jet $i$. Figure 5 shows, after background subtraction and some

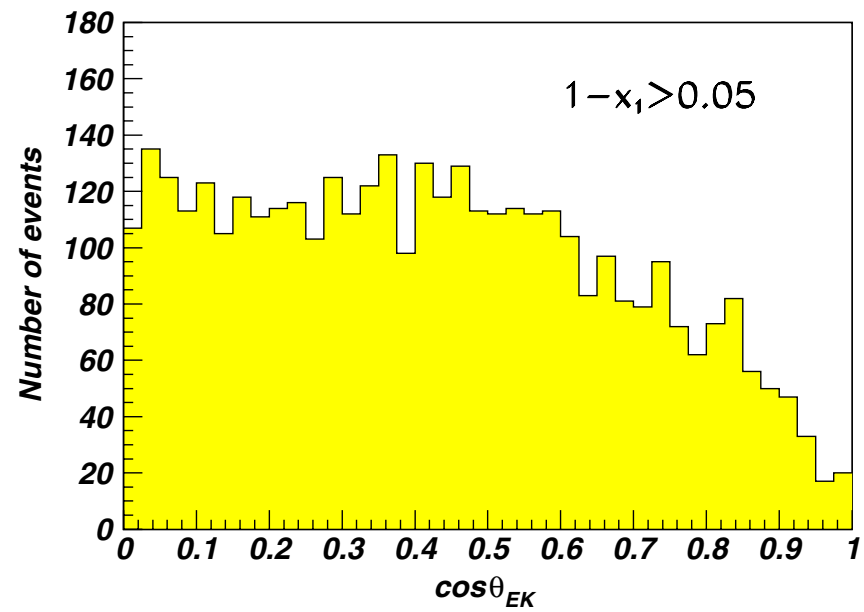

FIG. 5 (color online). Cosine of the Ellis-Karliner angle $\theta_{\mathrm{EK}}$ for 3-jet events selected at $\sqrt{s}=1 \mathrm{TeV}$ with the additional cut $1-x_{1}>0.05$. detector acceptance corrections, the cosine of the EllisKarliner angle distribution proposed to discriminate between the vector and scalar nature of the radiated $\gamma^{\prime}$. In order to avoid infrared divergences, the analysis is restricted to a region safely away from $x_{1}=1$, by the cut $1-$ $x_{1}>0.05$. Distinction between the vector and scalar particle interpretations is made only on the basis of the shape of the distribution: a spin-1 particle provides a flat behavior near $\cos \theta_{\mathrm{EK}}=0$, while a spin- 0 object yields a rising behavior [12]. Thus, spin-1 assignment for the paraphoton is highly favored over spin 0 . Alternatively, after interpreting a signal candidate event as a 3-jet event, the polar angle distribution of the normal to the three-jet plane, $\theta_{N}$, was proposed as a variable to distinguish between the vector and scalar hypothesis of the emitted particle [24,25]. We briefly discuss this variable, which is defined by the crossproduct of the two fastest jets as a function of a thrust cutoff $T_{C}$, in order to be able to establish that the parameter $\alpha_{N}$ extracted from the distributions is (i) independent of the thrust cutoff chosen and (ii) close to $-\frac{1}{3}$ as predicted for the spin- 1 interpretation of the $\gamma^{\prime}$. The cosine distributions of the angle $\theta_{N}$ are shown in Fig. 6 for various thrust cutoff values. The spectra, corrected for background and detector effects, were fitted to the expression

$$
\frac{1}{N} \frac{d N}{d \cos \theta_{N}}=\frac{1}{2\left(1+\frac{1}{3} \alpha_{N}\right)}\left(1+\alpha_{N} \cos ^{2} \theta_{N}\right)
$$
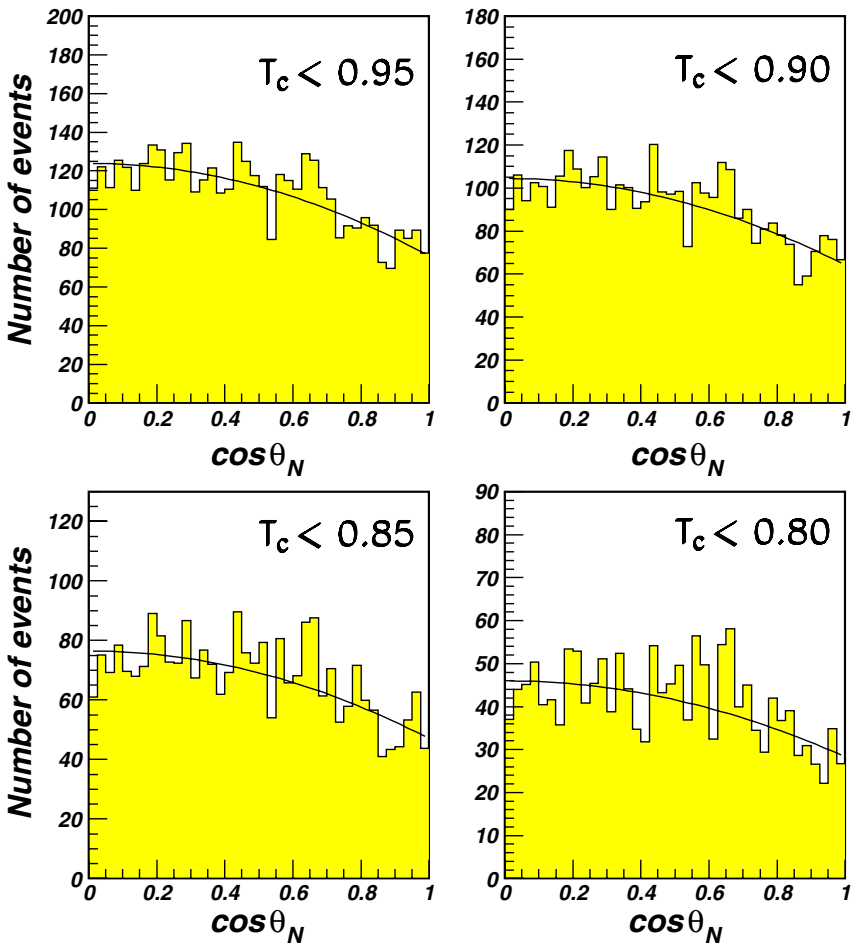

FIG. 6 (color online). Polar angle distributions of the normal to the three-jet plane for four different thrust cutoff values at $\sqrt{s}=$ $1 \mathrm{TeV}$. The curves represent the results of the fits described in the text. 
TABLE III. Values of the parameter $\alpha_{N}$ for the normal to the three-jet plane with $T<T_{C}$ at $\sqrt{s}=1 \mathrm{TeV}$.

\begin{tabular}{cc}
\hline \hline$T_{C}$ & $\alpha_{N}$ \\
\hline 0.95 & $-0.386 \pm 0.036$ \\
0.90 & $-0.382 \pm 0.039$ \\
0.85 & $-0.385 \pm 0.045$ \\
0.80 & $-0.384 \pm 0.058$ \\
0.75 & $-0.411 \pm 0.072$ \\
\hline \hline
\end{tabular}

predicted for vector particle emission [26] with $\alpha_{N}=-\frac{1}{3}$. Good agreement between data and the theoretical expectation is found. In particular, as seen from Table III, the $\alpha_{N}$ values are found to be independent of the thrust cutoff and quite close to $-\frac{1}{3}$, despite neglecting detailed correction factors in the analysis.

\section{CONCLUSIONS}

Some realistic extensions of the standard model suggest the existence of a new massless neutral gauge boson, denoted as the paraphoton $\gamma^{\prime}$ in this study. This particle is similar to the ordinary photon, but the interactions of the $\gamma^{\prime}$ are very distinct: couplings to SM fermions are proportional to fermion masses and therefore strongest to the top quark, and grow with the $\gamma^{\prime}$ momentum. Hence, paraphoton radiation off the top at the ILC is studied as the most promising process for $\gamma^{\prime}$ observation. Only the allhadronic top decay mode was selected to ensure a high signal-to-background ratio and to avoid complications due to final-state neutrinos in leptonic $W$ decays. A multivariate search strategy was used to better separate the signal from background. For a $5 \sigma$ paraphoton discovery significance, $e^{+} e^{-}$collisions at $1 \mathrm{TeV}$ allow us to bound the $\gamma^{\prime}$-top quark inverse coupling to $M / \sqrt{c_{t}} \lesssim 0.61 \mathrm{TeV}$, which is expected to be the most stringent limit accessible at the next generation colliders. For the sake of demonstration, two angular variables, the Ellis-Karliner angle and the polar angle of the normal to the $t \bar{t} \gamma^{\prime}$ plane, were studied to establish the vector nature of the $\gamma^{\prime}$. Both angular distributions are in accord with the spin-1 assignment of the paraphoton and inconsistent with e.g. a scalar hypothesis.

\section{ACKNOWLEDGMENTS}

The work of E. B. and V. B. is partly supported by Grant No. NS.1685.2003.2 of the Russian Ministry of Education and Science, and by Grant Nos. 08-02-91002 and 08-0292499 of the Russian Foundation for Basic Research. V. B. also acknowledges support from a grant of the "Dynasty" Foundation. E. B. and V.B. are grateful to DESY and Fermilab for their kind hospitality. We thank Bogdan Dobrescu for valuable discussion and reading the manuscript.
[1] B. Holdom, Phys. Lett. 166B, 196 (1986); 178, 65 (1986).

[2] L. B. Okun, Zh. Eksp. Teor. Fiz. 83, 8921 (1982) Sov. Phys. JETP 56, 502 (1982); L. B. Okun, M. B. Voloshin, and V. I. Zakharov, Phys. Lett. 138B, 115 (1984); A. Y. Ignatiev, V. A. Kuzmin, and M. E. Shaposhnikov, Phys. Lett. 84B, 315 (1979).

[3] H. Goldberg and L. J. Hall, Phys. Lett. B 174, 151 (1986); M. I. Dobroliubov and A. Y. Ignatiev, Phys. Rev. Lett. 65, 679 (1990); R. N. Mohapatra and I.Z. Rothstein, Phys. Lett. B 247, 593 (1990).

[4] B. A. Dobrescu, Phys. Rev. Lett. 94, 151802 (2005).

[5] M. Pospelov and A. Ritz, Phys. Rev. D 63, 073015 (2001).

[6] The ILC reference design report (RDR) is available from http://www.linearcollider.org/cms/.

[7] A. Pukhov et al., Report No. INP-MSU 98-41/542, arXiv: hep-ph/9908288; E. Boos et al., Nucl. Instrum. Methods Phys. Res., Sect. A 534, 250 (2004).

[8] T. Sjostrand, L. Lonnblad, S. Mrenna, and P. Skands, arXiv:hep-ph/0308153.

[9] J. A. Aguilar-Saavedra et al., arXiv:hep-ph/0106315.

[10] E. Boos et al., arXiv:hep-ph/0109068.

[11] M. Pohl and H. J. Schreiber, arXiv:hep-ex/0206009.
[12] R. Brandelik et al., Phys. Lett. 97B, 453 (1980); S. Wu, Phys. Rep. 107, 59 (1984).

[13] H. J. Behrend et al., Phys. Lett. 110B, 329 (1982).

[14] Ch. Berger et al., Phys. Lett. 97B, 459 (1980).

[15] D. Burger et al., Proceedings of the 21st Internationale Conference on High Energy Physics, Paris, 1982; J. Phys. (Paris) 43, C3 (1982).

[16] G. Alexander et al., Z. Phys. C 52, 283 (1991).

[17] B. Adeva et al., Phys. Lett. B 263, 551 (1991).

[18] P. Abreu et al., Phys. Lett. B 274, 498 (1992).

[19] J. Ellis and I. Karliner, Nucl. Phys. B148, 141 (1997).

[20] W. Braunschweig et al., Z. Phys. C 36, 349 (1987).

[21] J.del Peso, Ph.D. Thesis, Universad Autonoma de Madrid, [Report No. FTUAM-EP-89-02 (unpublished)].

[22] M. Althoff et al., Z. Phys. C 29, 29 (1985).

[23] W. Braunschweig et al., Phys. Lett. B 214, 286 (1988).

[24] G. Kramer, G. Schierholz, and J. Willrodt, Phys. Lett. 79B, 249 (1978); 80B, 433 (1978); Z. Phys. C 4, 149 (1980).

[25] K. Koller et al., Z. Phys. C 6, 131 (1980).

[26] J. Koerner, G. A. Schuler, and F. Barreiro, Phys. Lett. B 188, 272 (1987). 